\title{
Sensometrics: Identifying Pen Digitizers by Statistical Multimedia Signal Processing
}

\author{
Andrea Oermann, Claus Vielhauer and Jana Dittmann \\ Otto-von-Guericke University of Magdeburg \\ ITI Research Group \\ Postfach 4120 \\ 39016 Magdeburg \\ Germany
}

\begin{abstract}
In this paper a new approach will be introduced to identify pen-based digitizer devices based on handwritten samples used for biometric user authentication. This new method of digitizer identification based on their signal properties can also be seen as an influencing part in the new research area of so-called sensometrics. The goal of the work presented in this paper is to identify statistical features, derived from signals provided by pen-based digitizer tablets during the writing process, which allow identification, or at least group discrimination of different device types. Based on a database of a total of approximately 40,000 writing samples taken on 23 different pen digitizers, specific features for class discrimination will be chosen and a novel feature vector based classification system will be implemented and experimentally validated. The goal of our experimental validation is to study the class space that can be obtained, given a specific feature set, i.e. to which degree single tablets and/or groups of pen digitizers can be identified using our developed classification by a decision tree model. The results confirm that a group discrimination of devices can be achieved. By applying this new approach, the 23 different tablets from our database can be discriminated in 19 output groups.
\end{abstract}

\section{Motivation}

Biometrics has become a household word and now sensometrics is also gaining increasing importance. The word sensometrics has its roots in the Latin word sensere, which means "to feel with one's senses". The word sensor derives from the same origin. Sensometrics in itself basically refers to mathematical and statistical methods for the analysis of the sensory and consumer science and preference data [1]. We now introduce sensometrics in the field of computer science and digital multimedia signal processing. We define sensometrics as the application of methods for the analysis and determination of a particular sensor (device) an original digital sample is sampled with, whereby the actual context in which the original sampling has been performed can vary. For example, for identifying digital cameras, any photographic image can be taken into account, whereas for identifying pen digitizer, sensors for capturing handwriting samples such as signatures can be analyzed.

At least two main applications for sensometrics can be identified today: forensics and biometrics. While forensics refers to the posterior detection and proofing of tracks entities left behind, which generally plays a role in a criminalistic context where evidence is needed in court, biometrics is the on-line identification and verification of an individual. Instead of identifying a person by external information such as user knowledge (passwords, PINs), a physical key, smartcards or special token, which can be lost, stolen or handed over, a biometric system identifies a person itself based on its given characteristics. The advantage of biometric user authentication is the unique and reliable identification and verification of a human being's identity based on his/her individual biometrical attributes such as speech and handwriting as behavioral-based modalities and fingerprint, face, iris, retina, or hand geometry as physiological modalities. However, the accuracy of biometric methods depends on a number of system parameters, one important aspect here is sensor characteristics. For the particular modality of online handwriting, it has been shown recently, that the recognition performance may vary significantly for different handwriting tablets [2]. Furthermore, integrity of biometric reference data is an important aspect in authentication system design. Here, one of the important aspects is the

Multimedia on Mobile Devices 2007, edited by Reiner Creutzburg, Jarmo Takala, Jianfei Cai, Proc. of SPIE-IS\&T Electronic Imaging, SPIE Vol. 6507, 65070I,

(C) 2007 SPIE-IS\&T $\cdot 0277-786 X / 07 / \$ 18$ 
question whether or not stored reference data originates from a specific sensor. For both aspects, the application of sensometrics in biometric recognition systems may enable the detection and validation of the original sensor type in the future and may improve the level of security in infrastructures and applications. Therefore, sensometrics connects biometrics and forensics.

Also, regarding the increasing demand of mobile multimedia devices, identifying pen digitizers can be of much benefit in order to assure a higher security depending on the corresponding applications. Handwriting, especially the signature, seems to be one of the most acceptable and reliable method to easily provide identities by biometrics even in the digital age, e.g. on pen-based mobile devices. One idea is to additionally provide information about the used device. For example, regarding mobile payment, identifying not only the signature but also the used mobile device as a double check of the person could increase the security significantly.

There are already approaches existing for categories of sensors other than pen digitizers such as for camera, microphone and printer identification. In [3] and [4] an approach of using the sensor's pattern noise for digital camera identification is presented, which is extracted from the images using a wavelet-based denoising filter. Thus, they have been able to identify the correct camera out of 9 cameras, including two cameras of exactly the same model, without a single misclassification for several thousand images even from images that underwent subsequent JPEG compression and/or resizing. Further, with a forensic background, a concept for classifying and analyzing audio is presented in [5] in order to determine the microphone and the authenticity of a speaker's environment. Therefore, a so-called Verifier-Tuple is introduced, which as a scalable method enables a detailed analysis and classification of every kind of media. An approach for identifying printer based on graylevel co-occurrence features is presented in [6], while another approach for the forensic identification of printers based on svm techniques is presented in [7]. An overview of methods for forensic characterization of devices in given in [8], where current forensic identification techniques for RF devices, printers, and cameras are presented and examined, and a generalization for the use with other devices is shown. In this paper, an approach for identifying pen digitizers based on the captured handwritten samples is provided. A feature vector based classification in form of a decision tree model is developed in order to differentiate pen digitizer devices.

This paper is structured as follows. In section 2, an overview about the general biometric handwriting recognition, sampling and data representation is presented. Pen digitizers and their characteristics are briefly introduced in section 3. In section 4, our new concept for identifying pen digitizers will be explained. In section 5 , the test environment and test set the will be outlined. Based on the presented concept in section 4, the used test methodology to identify a pendigitizer as well as the test goals will also described in this section 5. In section 6 , the test results will be presented and discussed. The paper closes with a conclusion and an outlook in section 7.

\section{General Biometric Handwriting Recognition, Sampling and Data Representation}

The characteristics of the generation of a particular handwriting can be specified by the movement of the pen tip during the writing process. The main dimensions of this movement are pen position (horizontal/vertical), pen tip pressure and pen angles. Digitizer tablets provide sensor technology for the analog-digital conversion of these kinds of dynamics. Today, digitizer tablets are becoming increasingly common, mainly due to the wide distribution of pen-enabled computers such as PDA or Tablet PCs. These types of computers provide position information, represented as sequences of pen position points at discrete and continuous time intervals.

This representation of continuous information is also denoted as sampled signals, and for the case of position signal, we use the notation $x(t)$ for horizontal pen position signals and $y(t)$ for vertical pen position signals. The spatial resolution of the pen position signal is a characteristic property of the digitizer tablet. The pen tip pressure signal is denoted as $p(t)$. Finally, some digitizer tablets today provide pen azimuth signals, denoted as $\Theta(t)$, the orientation of the vertical projection of the pen onto the writing surface, similar to a compass, as well as pen altitude signals $\Phi(t)$, describing the angle of the pen above the writing surface. A classification of pen digitizers regarding their physical parameters will be provided in section 3 .

Figure 1 illustrates the process of sampling the handwriting position signals into sequences of discrete values. The goal of biometric user authentication using handwriting is the determination of similarities based on the application of these sampled signals. 


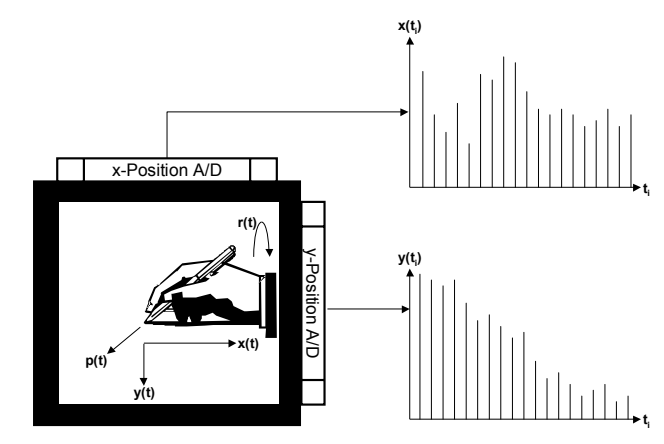

Fig. 1. Digitized handwriting data sampling and representation (from [2])

The biometric user authentication process is generally structured as follows: Reference data is sampled within an enrollment and stored in a relevance database. For the case of handwriting biometrics, signals are sampled and analyzed for authentication. Authentication algorithms, as described for example in [9], [10], and [11], consider certain parameters of the reference storage. Well known algorithms are for example Dynamic time Warping (DTW), HiddenMarkov-Models (HMM), Neural Networks, Multi Level Approaches, or BioHash [12]. Two different goals of authentication can be outlined. The first goal is the verification of one particular known user of the reference storage and refers to a comparison of one signal sampling to one particular reference storage sampling (1:1 comparison). The second goal is the identification of a particular not known user which indicates a comparison of one particular signal sampling to $n$ reference storage samplings (1:n comparison), whereby $n$ denotes the overall number of subjects actually enrolled to the given system. Depending on the desired authentication mode, the system parameters may change.

In order to provide a sufficient measurement quantity for evaluating biometric user authentication algorithms, so-called semantics are needed. Semantics refers to different user specific writing content such as e.g. signature, PIN, symbols, and phrases [2]. Although semantics are important in the field of biometrics, it is not influencing the sensometrics approach of identifying the used pen digitizer presented in this paper. Therefore, a detailed understanding of the impact of different semantics is not required in the context of sensometrics and we confine to explaining the important aspects for this paper.

\section{Pen Digitizers and their Physical Characteristics}

As described in detail in [2], pen digitizers have certain characteristic features given by their physical parameters. Thus, a general classification can easily be realized by analyzing these physical parameters.

The first physical parameter is the spatial resolution measured in lines per inch (lpi). It indicates the resolution of the horizontal and vertical pen position signal. For some digitizers the spatial resolution is limited by the screen resolution, while for others, in particular those, where the digitizer resolution is identical to its screen resolution, it is impacted by the digitizer's basic physical design. For a third class of digitizers, the spatial resolution varies depending on the device driver and achieves a higher resolution. Consequently, three categories regarding the spatial resolution can be outlined according to [2]: low (<100 lpi), medium (100 lpi $\leq$ resolution $<2000$ lpi) and high resolution ( $\geq 2000$ lpi). A second physical parameter indicates the pressure signal quantization based on the signal dimensionality. The pressure signal quantization refers to a digitizer's ability of capturing the amount of pressure to its surface generated during a users writing sequence. Two categories are differentiated, a binary pressure quantization (PenUp/PenDown) and pressure quantization $>2$. A third physical parameter indicates the pen angle signals azimuth and altitude which are either provided by a digitizer or not. A detailed description of this classification can be found in [2].

\section{Identifying Pen Digitizers by Feature Analysis}

In this section, we introduce the basic procedure for identifying pen digitizers. Thus, we first outline the parts of the procedure. Further, the required parameters for feature extraction are described into detail in order to finally present the used feature vector as the fundamental contribution for our decision tree model to classify pen digitizers. 


\subsection{Identifying Pen Digitizers - Procedure}

After data acquisition, certain techniques are applied in preprocessing in order to extract particular features. We differentiate between two main steps: First, the analyzing and following the testing. The idea of the general approach can be introduced as follows: First, the data is analyzed in order to find the decision tree model as the basis for classification. We then test the classification with our database by matching extracted features of specific devices to the decision tree model. Thus, a decision can be made and as the outcome the device the tested sample is captured with is classified to one of the predefined output classes (OPC). The complete procedure can be followed in Figure 2.

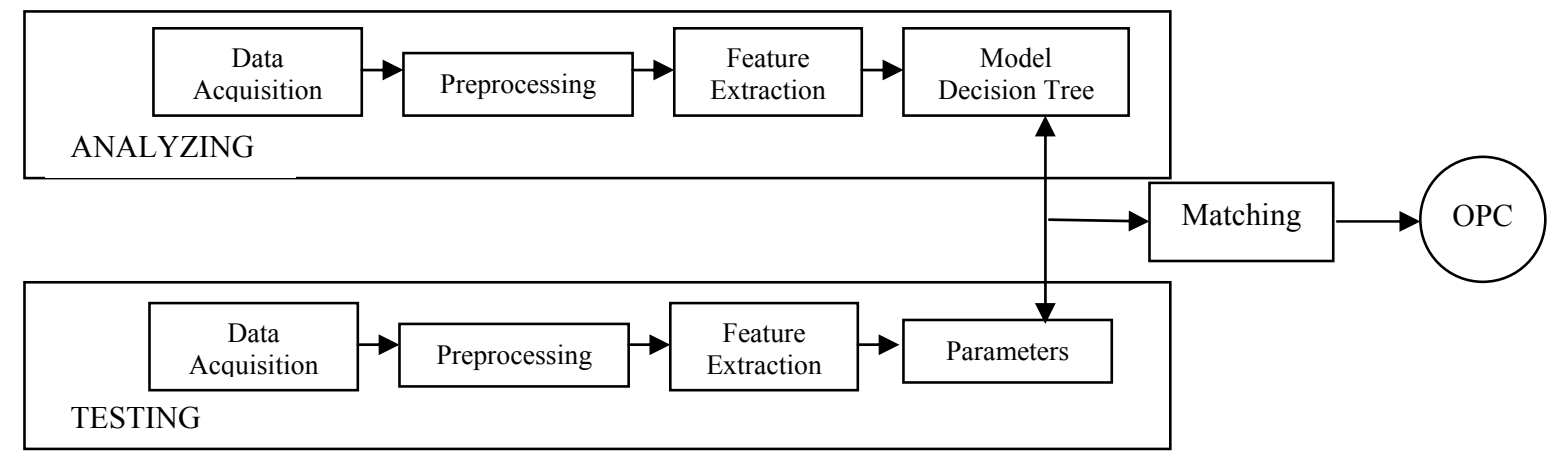

Fig. 2. Procedure of identifying pen digitizers

\subsection{Data Acquisition: Sampled Data Parameters and Input Vector}

As generally introduced in section 2, certain parameters are determined for each sampling point. In our tests the following particular parameters are considered: x-position $x(t)$, y-position $y(t)$, old x-position $x \_o l d(t)$, old y-position $y \_$old $(t)$, timestamp $t$ in $m s$, pressure $p(t)$, azimuth $\Theta(t)$, and altitude $\Phi(t)$. According to its resolution, positive integers are returned by the corresponding device. The old x-position and y-position are the coordinates of the previous sampling point. For the first sampling point this is denoted by $(-1,-1)$. The timestamp $t$ indicates the time in milliseconds at which a contact is made at a particular $(x, y)$ coordinate. The timer starts with a value of 0 for the starting of a writing sequence and is measured in milliseconds. Thus, the timestamp value is always relative to a sample. Most pen digitizers have different pressure quantization levels: no pressure (0), 128, 256, 512, or 1024.

The described parameters can be summarized in a so-called input vector $I$ as presented in equation 1 .

$$
I=\left\{\text { Sample Id }, \text { Event Id }, x(t), y(t), x \_ \text {old }(t), y \_o l d(t), t, p(t), \Theta(t), \Phi(t)\right\}
$$

Sample_Id and Event_Id denote a unique identifier tuple for a connected handwriting sequence within our database. Since feature vectors are calculated for each sample, Sample Id, which is unique to each sample, is used to discriminate the samples while Event Id refers to the semantics. The coordinates $(x(t), y(t))$ and $\left(x \_\right.$old $(t), y \_$old $\left.(t)\right)$ are directly retrieved from the database. Starting points as well as pen-up and pen-down positions are identified by "-1" at $(x(t), y(t))$ and $\left(\left(x \_\right.\right.$old $(t), y \_$old $\left.(t)\right)$ coordinates respectively. Thus, a combination of $\left(\left(x \_o l d(t), y \_o l d(t)\right)\right.$ coordinate couples is considered for calculating the features.

\subsection{Preprocessing and Feature Extraction}

Preprocessing refers to modifying the raw data in order to obtain normalized data points for subsequent processing. Hereby, aberrant and unwanted data is removed. Because users of pen digitizers usually do not continuously write on the screen, but interrupt their flow of writing by lifting the pen, the total time of writing is measured by excluding the time of interruptions within preprocessing. Thus, only the time the pen actually hits the device is considered and all values above a defined threshold are removed. This preprocessing is necessary for calculation of efficient feature vectors. Further, all time difference values above 100 milliseconds are generally eliminated in preprocessing. This is motivated by the observation that most devices do not have sampling rates less than $10 \mathrm{~Hz}$ leading us to ignore the higher values. 
After preprocessing, the feature extraction is performed. Particular features are extracted in order to identify crucial patterns of a sample, which indicate the particular device used for capturing the sample. These features will be described in detail in the coming subsections. In summary, the feature set is composed of attributes altitude type $A T$, pressure level type $P L$, pressure difference value $P D$, time difference value $T D$, and average sampling rate $A V$. In addition, the statistical feature maximum pressure $\max (p(t))$ needs to be calculated in order to determine the attributes of the feature vector. Later in section 4.4., we will outline how a feature vector based on these features is composed.

\subsubsection{Altitude type (AT)}

Based on the samples in our database, 3 different types of altitude values can be differentiated, a zero altitude value for low end devices, a constant value of 900 for medium level devices and a variable altitude value for high level devices. These three values $\{0,900$, Variable $\}$ are mapped to $\{1,2,3\}$ for decision.

\subsubsection{Pressure Level Type (PL)}

The pressure is one of the attributes returned by the device. The number of pressure levels a device can support is calculated by using maximum pressure $\max (p(t))$ of a sample. High end devices generally support 1024 pressure levels while some devices support a variety of 512, 256, 128 pressure levels, and other devices don't calculate pressure at all. These values are mapped to $\{1,2,3,4,5\}$ for decision.

\subsubsection{Maximum Pressure $\max (p(t))$}

Based on the pressure value detected for each sample point, the maximum pressure value $\max (p(t))$ is calculated for each sample in order to determine the number of pressure levels a device supports. From the data it is observed that for a device having $n$ pressure levels, the maximum pressure value of a particular sample is typically $\geq N / 2$. For example, if a device can detect 512 pressure levels then the maximum pressure of any sample of that device is typically $\geq 256$. Hence, half the maximum pressure value is taken as a threshold.

\subsubsection{Pressure Difference Value (PD)}

Assuming, pressure values of two subsequent sample points do not show a high degree of variation, the pressure difference between subsequent sample points is considered as an attribute in the feature vector for detecting the device type. By doing so, device specific patterns can be determined. A pressure difference value is calculated as presented in equation 2.

$$
P D=|p(t)-p(t+1)|
$$

Considering the consecutive pressure difference values of all tested samples, three different pressure difference patterns could be identified, as presented in Table 1, Table 2, and Table 3. Each row indicates a sample, and the values represent a histogram of pressure difference values of consecutive sample points. For example, pressure difference value 0 is occurring 33 times in the first sample in table 1, while in table 2 and table 3 pressure difference value 0 is occurring 122 times for the first sample. Due to space constraints only the number of pressure difference values from 0 up to 7 subsequent sample points is shown. In the pattern descriptions "NNNNNNNN", "N0N0N0N0", and "NX00NX00", N denotes significant pressure differences occurring, 0 denotes no pressure differences occurring, and $\mathrm{X}$ denotes minor pressure differences occurring with $\approx \mathrm{N} / \mathrm{X} \geq 10$.

Table. 1. Pattern1: NNNNNNNN
\begin{tabular}{|l|l|l|l|l|l|l|l|}
\hline $\mathbf{0}$ & $\mathbf{1}$ & $\mathbf{2}$ & $\mathbf{3}$ & $\mathbf{4}$ & $\mathbf{5}$ & $\mathbf{6}$ & $\mathbf{7}$ \\
\hline 33 & 27 & 22 & 23 & 19 & 12 & 13 & 5 \\
\hline 18 & 9 & 20 & 6 & 6 & 17 & 5 & 2 \\
\hline 211 & 8 & 16 & 11 & 3 & 10 & 7 & 4 \\
\hline 216 & 17 & 9 & 6 & 5 & 12 & 4 & 2 \\
\hline
\end{tabular}

Table. 2. Pattern 2: NONONONO

\begin{tabular}{|l|l|l|l|l|l|l|l|}
\hline $\mathbf{0}$ & $\mathbf{1}$ & $\mathbf{2}$ & $\mathbf{3}$ & $\mathbf{4}$ & $\mathbf{5}$ & $\mathbf{6}$ & $\mathbf{7}$ \\
\hline 122 & 0 & 81 & 0 & 93 & 0 & 77 & 0 \\
\hline 94 & 0 & 77 & 0 & 68 & 0 & 71 & 0 \\
\hline 54 & 0 & 72 & 0 & 54 & 0 & 56 & 0 \\
\hline 67 & 0 & 55 & 0 & 55 & 0 & 43 & 0 \\
\hline
\end{tabular}

Table. 3. Pattern 3: NX00NX00

\begin{tabular}{|l|l|l|l|l|l|l|l|}
\hline $\mathbf{0}$ & $\mathbf{1}$ & $\mathbf{2}$ & $\mathbf{3}$ & $\mathbf{4}$ & $\mathbf{5}$ & $\mathbf{6}$ & $\mathbf{7}$ \\
\hline 122 & 1 & 0 & 0 & 106 & 4 & 0 & 0 \\
\hline 190 & 2 & 0 & 0 & 110 & 3 & 0 & 0 \\
\hline 226 & 1 & 0 & 0 & 113 & 10 & 0 & 0 \\
\hline 418 & 1 & 0 & 0 & 82 & 9 & 0 & 0 \\
\hline
\end{tabular}

In pattern1 (NNNNNNNN), all pressure difference values can be determined, which indicates a device's raised pressure sensitivity. In pattern 2 (N0N0N0N0), exclusively even and no odd differences can be obtained. Pattern 3 (NX00NX00) refers to a specific sequence the determination of pressure difference values maintains: The number of the obtained 
pressure difference value 0 is quite high, while the following pressure difference value 1 is relatively low in comparison to its predecessor. The third and fourth difference values are zero. This sequence is repeating for the whole sample. In other words, this pattern indicates only few pressure difference values can be determined which leads to the assumption that only low resolution devices show this pattern. For the decision of the device these three patterns $\{$ NNNNNNNN, N0N0N0N0, NX00NX00 $\}$ are mapped to $\{1,2,3\}$ for decision.

\subsubsection{Time Difference Value TD}

Similar to pressure difference, the time differences between all subsequent sample points are determined. Because of the fact, that the shorter the time period between sampling two subsequent points, the higher the sampling rate, we assume determing the time difference values of a sample may characterize the used device. We observed that most devices do not have sampling rates less than $10 \mathrm{~Hz}$. Therefore, we choose to ignore the higher values. Thus, in preprocessing all time difference values above 100 milliseconds are generally eliminated. After calculating histograms of the time difference values, similar as it has been done for the pressure difference values, specific patterns could be detected. A lot of different time difference patterns could be acquired. We assigned six patterns as the most crucial ones for identifying the used pen digitizer. Hereby, the distribution of the values determines the pattern. Due to space constraints we only include two exemplary histograms of the time difference values (Table 4 and Table 5) in this paper and further but summarize all patterns.

Table. 4. Exemplary pattern 1: $(7,8,15,16)$

\begin{tabular}{|r|r|r|r|r|r|r|r|r|r|r|r|r|r|r|r|r|}
\hline $\mathbf{0}$ & $\mathbf{1}$ & $\mathbf{2}$ & $\mathbf{3}$ & $\mathbf{4}$ & $\mathbf{5}$ & $\mathbf{6}$ & $\mathbf{7}$ & $\mathbf{8}$ & $\mathbf{9}$ & $\mathbf{1 0}$ & $\mathbf{1 1}$ & $\mathbf{1 2}$ & $\mathbf{1 3}$ & $\mathbf{1 4}$ & $\mathbf{1 5}$ & $\mathbf{1 6}$ \\
\hline 0 & 0 & 0 & 0 & 0 & 0 & 0 & $\mathbf{2 8}$ & $\mathbf{1 1 0}$ & 0 & 0 & 0 & 0 & 0 & 0 & $\mathbf{3 2}$ & $\mathbf{5 4}$ \\
\hline 0 & 0 & 0 & 0 & 0 & 0 & 0 & $\mathbf{1 8}$ & $\mathbf{1 2 8}$ & 0 & 0 & 0 & 0 & 0 & 0 & $\mathbf{4 3}$ & $\mathbf{4 7}$ \\
\hline 0 & 0 & 0 & 0 & 0 & 0 & 0 & $\mathbf{3 5}$ & $\mathbf{1 1 1}$ & 0 & 2 & 1 & 0 & 0 & 0 & $\mathbf{2 4}$ & $\mathbf{6 2}$ \\
\hline 0 & 0 & 0 & 0 & 0 & 0 & 0 & $\mathbf{1 3}$ & $\mathbf{1 0 5}$ & 0 & 0 & 0 & 0 & 0 & 0 & $\mathbf{3 7}$ & $\mathbf{3 7}$ \\
\hline 0 & 0 & 0 & 0 & 0 & 0 & 0 & $\mathbf{3 1}$ & $\mathbf{8 9}$ & 0 & 0 & 0 & 0 & 0 & 0 & $\mathbf{1 8}$ & $\mathbf{5 5}$ \\
\hline 0 & 0 & 0 & 0 & 0 & 2 & 1 & $\mathbf{1 8}$ & $\mathbf{1 1 6}$ & 0 & 0 & 0 & 0 & 0 & 0 & $\mathbf{3 8}$ & $\mathbf{4 6}$ \\
\hline 0 & 0 & 0 & 0 & 0 & 0 & 0 & $\mathbf{2 5}$ & $\mathbf{1 1 1}$ & 0 & 0 & 0 & 0 & 0 & 0 & $\mathbf{3 1}$ & $\mathbf{5 2}$ \\
\hline 0 & 0 & 0 & 0 & 0 & 3 & 0 & $\mathbf{1 3}$ & $\mathbf{9 1}$ & 0 & 0 & 0 & 0 & 0 & 0 & $\mathbf{3 1}$ & $\mathbf{3 5}$ \\
\hline
\end{tabular}

Table. 5. Exemplary pattern 2: $(5,10)$

\begin{tabular}{|r|r|r|r|r|r|r|r|r|r|r|r|r|r|r|r|r|}
\hline $\mathbf{0}$ & $\mathbf{1}$ & $\mathbf{2}$ & $\mathbf{3}$ & $\mathbf{4}$ & $\mathbf{5}$ & $\mathbf{6}$ & $\mathbf{7}$ & $\mathbf{8}$ & $\mathbf{9}$ & $\mathbf{1 0}$ & $\mathbf{1 1}$ & $\mathbf{1 2}$ & $\mathbf{1 3}$ & $\mathbf{1 4}$ & $\mathbf{1 5}$ & $\mathbf{1 6}$ \\
\hline 0 & 0 & 5 & 0 & 0 & $\mathbf{3 2}$ & 0 & 0 & 0 & 0 & $\mathbf{3 7}$ & 0 & 0 & 0 & 0 & 0 & 0 \\
\hline 0 & 2 & 4 & 0 & 0 & $\mathbf{2 6}$ & 0 & 0 & 0 & 0 & $\mathbf{2 7}$ & 1 & 0 & 0 & 0 & 0 & 0 \\
\hline 0 & 2 & 4 & 0 & 0 & $\mathbf{3 5}$ & 1 & 0 & 0 & 0 & $\mathbf{3 8}$ & 0 & 0 & 0 & 0 & 0 & 0 \\
\hline 0 & 2 & 4 & 0 & 0 & $\mathbf{3 0}$ & 0 & 0 & 0 & 0 & $\mathbf{3 0}$ & 1 & 0 & 0 & 0 & 0 & 0 \\
\hline 0 & 2 & 5 & 0 & 0 & $\mathbf{2 6}$ & 0 & 0 & 0 & 0 & $\mathbf{2 8}$ & 1 & 0 & 0 & 0 & 0 & 0 \\
\hline 0 & 0 & 4 & 0 & 0 & $\mathbf{3 0}$ & 0 & 0 & 0 & 0 & $\mathbf{3 0}$ & 1 & 0 & 0 & 0 & 0 & 0 \\
\hline 0 & 2 & 4 & 0 & 0 & 44 & 2 & 0 & 0 & 0 & $\mathbf{4 6}$ & 1 & 0 & 0 & 0 & 0 & 0 \\
\hline 0 & 2 & 4 & 0 & 0 & $\mathbf{9 0}$ & 0 & 0 & 0 & 0 & $\mathbf{9 4}$ & 0 & 0 & 0 & 0 & 0 & 0 \\
\hline
\end{tabular}

Before summarizing the patterns, the term "mode" needs to be determined. We define a "mode" as the specific time difference value of the general time interval (time difference) between two subsequent sample points $n-1$ and $n$ of a sample. For one sample a time difference value can occur more than just once. Each column indicates a mode, while each row refers to a sample. Mode 7 for example is the time difference value 7, which in table 4 occurs 28 times for the first sample and in table 5 does not occur at all (0) for all samples. Depending on the specific sample, a mode can have different values. Depending on their physical parameters, pen digitizers show specific characterizing modes which can be summarized as patterns.

In pattern $a$, all modes $n$ are characterizing, which indicates that the time difference values are distributed throughout the whole sample. This mainly occurs in high end devices. In pattern $b$, the distribution of time difference values is characterizing in modes $7,8,15$, and 16 . In pattern $c$, modes 5 and 10 are the characterizing ones. Most of zero pressure devices show this pattern. In pattern $d$, modes 2 and 3 are characterizing. In pattern $e$, modes 3, 4, 5, and 10 are characterizing. In pattern $f$, modes 12,13 , and 14 are characterizing. Finally, these different patterns are mapped to the constant numeric attributes $\{1,2,3,4,5,6\}$ for the decision.

\subsubsection{Average Time Period AV}

The average time period is the mean time period between any two consecutive sample points of a sample. Because of the time period of two subsequent sample points does not remain constant in some devices, it can hardly be considered as a parameter for identifying the used pen digitizer. Therefore, we calculate an average of all these time periods. The average time period is determined as it is presented in equation 3.

$$
A V=\frac{1}{N} \sum_{i=1}^{N-1} t\left(x_{i+1}\right)-t\left(x_{i}\right)
$$


The inverse of the average time period results in the average sampling rate of the device. Most devices have a sampling rate of 100 , while some devices have a sampling rate of 125 , and others have a sampling rate of 400 . We consider two main average time periods: $A V=1$ indicates $5.5<A V<8.5$, while $A V=2$ refers to $8.5<A V<11$. Hence, the average time periods are mapped to $\{1,2\}$ for the decision.

\subsection{Feature Vector}

Considering all explained features, a feature vector is formally described as presented in equation 4 . It contains the attributes altitude type $A T$, pressure level type $P L$, pressure difference value $P D$, time difference value $T D$, and average sampling rate $A V$.

$$
F=\{A T, P L, P D, T D, A V\}
$$

Based on the explanations of the attributes presented in subsection 4.3, the attributes of the feature vector can have the following states, where 0 is added to capture unexpected values or outliers:

$\begin{array}{ll}\boldsymbol{A L} & \text { Altitude } \\ \boldsymbol{P L} & \text { Pressure level } \\ \boldsymbol{P D} & \text { Pressure difference } \\ \boldsymbol{T D} & \text { Time difference } \\ \boldsymbol{A} \boldsymbol{V} & \text { Average }\end{array}$

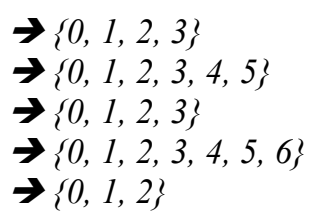

This feature vector containing the extracted features is then used in matching to compare a particular sample with the samples in the relevant data base in order to attribute the used device to a predefined output class (OPC).

\section{Test Environment and Test Set, Test Methodology and Test Goals}

In this section, we introduce the test environment and test set used for our studies. We further describe our test methodology with respect to the decision tree based classification and the resulting output classes. Further, test goals for our evaluation are defined at the end of this section.

\subsection{Test Environment and Test Set}

As the test environment we used PlataSign, which is a software tool developed by our research group in order to evaluate biometric user authentication systems, in particular those systems based on handwriting. Thus, we have been able to access already existing databases collected for earlier research tasks in the field of biometrics as presented in [2]. Because of this, a large test set of around 40,000 samples out of a number of data sets could be considered for testing. For the tests, presented in this paper, we consider samples taken with 23 different pen digitizers. Further, for each digitizer type, we have divided the database into two disjoint partitions: first, the analyzing partition for development of the decision tree model and the output classes (OPC) and second, a disjoint testing partitions for our subsequent evaluation. Partitioning of data into these two sets has been performed in a manner that for each digitizer type an equal number of samples is represented in either of the sets.

\subsection{Test Methodology and Test Goals}

The test methodology is based on the procedure of identifying pen-digitizers based on feature vectors extracted from the digitizer signals as described in section 4 , which are classified by a decision tree. For each of the decision nodes of the tree, we have derived the decision criteria based on an a-priori analysis for each digitizer category of the analysis portion of the database. Figure 3 presents the resulting decision tree.

The classification in the tree is performed for a given feature vector in a root-to-leaf manner. Initially at root level, any feature vector represents the total number of 23 candidate devices a specific device potentially can be classified to. At this first level, the first attribute (component AL, altitude) of the feature vector is applied as classification criterion into the three sub-sets Set A, B and C. On the second level, classification is done based on the attribute for pressure level (PL), then in the third level for pressure difference (PD), in the fourth step for time difference (TD) and finally in the lowest level (if applicable for a given feature vector) for average time period (AV). At the end of the classification process, the actual feature can be allocated to one output class. Note that each of the output classes may represent 
multiple digitizer types and further, pen-digitizers may be attributed to more than one output class. The actual output classes we achieved during the analysis phase will be discussed in the following subsection.

This test methodology of building up a decision tree model, predefining output classes and applying a sequence of parameters in order to enforce a decision in each step of the tree is our initial approach to identify a pen digitizer.

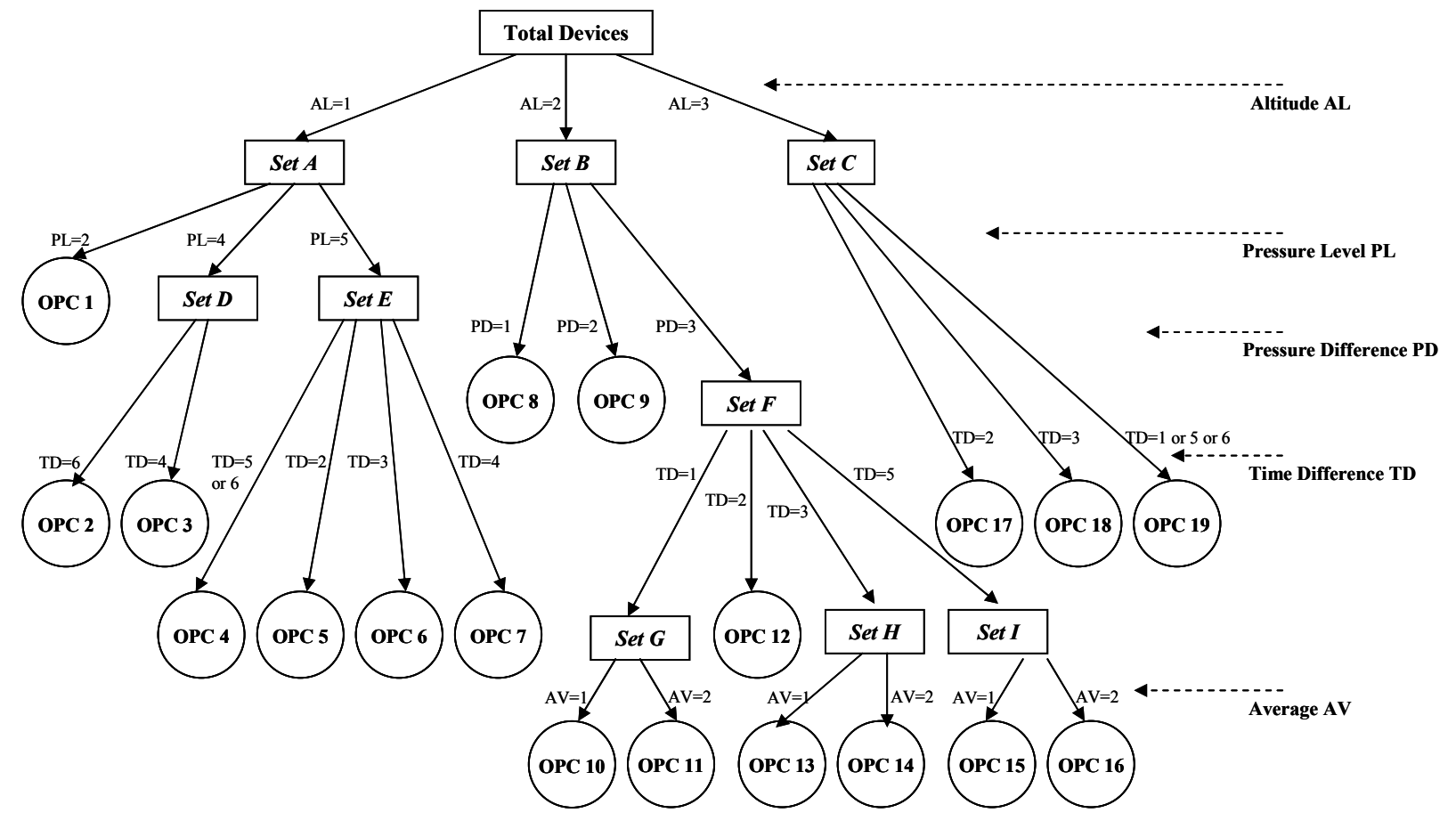

Fig. 3. Decision tree for the differentiation of 19 output classes (OPC)

\subsection{Output Classes OPC}

After the design of the decision tree based on the feature set components, we have determined the output classes from the analyzing set. For this purpose, we have performed classification for each of the approximately 20,000 samples in the analyzing partition of our database and registered, which digitizers have been classified in which of the 19 OPCs.

Table. 6. Output Classes (OPC)

\begin{tabular}{|l|l|}
\hline Output Classes & Pen Digitizers \\
\hline OPC 1 & Aiptec 8000 \\
\hline OPC 2 & Logitech IO \\
\hline OPC 3 & StepOver+Pad, StepOver+Pad Inkpen, StepOver blueM Pad \\
\hline OPC 4 & Wacom USB Screen \\
\hline OPC 5 & Intuos 2 Screen,Volito 2 Screen \\
\hline OPC 6 & Palm, Touch Eizo, Wacom Screen, Wacom USB Screen \\
\hline OPC 7 & Stepover No Pressure \\
\hline OPC 8 & Cintiq15x,Wacom USB No Angle, Toshiba \\
\hline OPC 9 & Wacom USB NO Angle \\
\hline OPC 10 & Toshiba \\
\hline OPC 11 & Wacom Volito \\
\hline OPC 12 & Wacom Volito \\
\hline OPC 13 & Toshiba, Pocket PC \\
\hline OPC 14 & Touch, Wacom USB NO Angle \\
\hline OPC 15 & Toshiba \\
\hline OPC 16 & Touch \\
\hline OPC 17 & Wacom USB, Wacom, Intuos 2 No Angle, Intuos \\
\hline OPC 18 & Intuos 2 No Angle, Intuos 2 Ink Pen, Wacom Intuos 2 \\
\hline OPC 19 & Wacom USB, Wacom Intuos 2 \\
\hline
\end{tabular}


The resulting memberships of individual digitizers in OPCs are shown in Table 6. Note that some output classes contain more than one device and some devices are represented in multiple OPCs. Thus, with our decision tree approach, we can so far partially identify individual digitizers, whereas in some cases only groups of tablets can be discriminated.

\subsection{Test Goals}

The goal of our experimental evaluation is to determine, to which degree a correct classification based on the decision tree model introduced in section 5.2 into the 19 output classes, as identified in the previous subsection, can be achieved. To do so, we have applied our classification scheme to the second portion of the test database, performed a second classification for approximately 20,000 samples, and registered the resulting OPC as well as the original tablet types. Test measurements in our experiments have been the Correct Identification and False Identification rates. The first rate denotes the ratio of number of correct classification and the total number of samples for each tablet category, whereas the False Identification rate represents the ratio between incorrect classifications for a given category and its total number of samples. In case of non-classifications, we further measure the number of non-classifications (i.e. due to erroneous program execution) in relation of the total number of samples in each category. The discussion of these measurements should allow for a first assessment to which degree the proposed scheme can perform robust identification of devices.

\section{Experimental Results}

Following the described test methodology in section 5 and the presented concept in section 4, the results of our experiments are presented as a matrix in Table 7. The table shows the comparison of the 23 pen digitizers in the rows and the observed classifications into 19 output classes in the columns. OPC0 is an additional output class and contains the outliers, i.e. non-classified samples which occurred due to program execution errors in our prototypical evaluation. Since the relative numbers of such non-classification occurrences is relatively low, we assume that they do not impact the overall tendency observed. Numbers printed in bold indicate absolute numbers of Correct Identifications, whereas italic numbers refer to the False Identifications of devices. Column "Total" summarizes the total number of tested devices for each device type while column "Average" presents the average positive identification rate in percent. In Table 8, a summarization of Correct Identification, False Identification and Non Classification ratios for each digitizer category is shown.

Table. 7. Distribution of devices in output groups after applying the feature vector

\begin{tabular}{|c|c|c|c|c|c|c|c|c|c|c|c|c|c|c|c|c|c|c|c|c|c|c|}
\hline \begin{tabular}{|l} 
Device/Output \\
\end{tabular} & OPC0 & OPC1 & OPC2 & OPC3 & OPC4 & OPC5 & OPC6 & OPC7 & OPC8 & OPC9 & OPC10 & OPC11 & OPC12 & OPC13 & OPC14 & OPC15 & OPC16 & \begin{tabular}{|l|l|} 
OPC17 \\
\end{tabular} & OPC18 & OPC19 & \begin{tabular}{|l|} 
Total \\
\end{tabular} & Average \\
\hline Toshiba Portégé Tablet-PC & 87 & & & & & & & & 1062 & & 1421 & 55 & & 573 & & $\begin{aligned} 41 \\
\end{aligned}$ & & & & & 3239 & $95.62 \%$ \\
\hline \begin{tabular}{|l|} 
Wacom Cintiq 15 \\
\end{tabular} & 117 & & & & & 31 & & & 10221 & 35 & & & 70 & & & & & & & & 10474 & $95.11 \%$ \\
\hline Wacom Intuos 2 & 252 & & & & & & 1619 & & 10 & 7 & & & & & & & & 2364 & 787 & 1589 & 6628 & $71.51 \%$ \\
\hline PocketPC & & & & & & & & & & & & & & 5 & & & & & & & 5 & $100 \%$ \\
\hline \begin{tabular}{|l} 
Logitech IO-Pen \\
\end{tabular} & 29 & & 3888 & & & & & & & & & & & & & & & & & & 3917 & $99.26 \%$ \\
\hline Wacom & 74 & & & & & & & & & & & & & & & & & 959 & & & 1033 & $92.84 \%$ \\
\hline Palm & & & & & & & 1512 & & & & & & & & & & & & & & 1512 & $100 \%$ \\
\hline Wacom USB & 7 & & & & & & 1866 & & & & & & & & & & & 1222 & & 260 & 3355 & $44.17 \%$ \\
\hline \begin{tabular}{|l} 
Touch \\
\end{tabular} & 27 & & & & 170 & & 1702 & & & & & & & & 640 & 18 & 504 & & 40 & 8 & 3109 & $36.8 \%$ \\
\hline Touch EIZO & & & & & & & 2050 & & & & & & & & & & & & & & 2050 & $100 \%$ \\
\hline \begin{tabular}{|l} 
Wacom Volito \\
\end{tabular} & & & & & & & & & 24 & & & 146 & 1216 & & & & & & & & 1386 & $98.27 \%$ \\
\hline Aiptec Hyperpen 8000 & & 45 & 33 & & & & & & & 9 & & & & & & & & & & & 87 & $51.72 \%$ \\
\hline Wacom Intuos 2 Inkpen & & & & & & & & & & & & & & & & & & & 214 & & 214 & $100 \%$ \\
\hline StepOver +Pad & & & & 210 & & & & & & & & & & & & & & & & & 210 & $100 \%$ \\
\hline StepOver +Pad Inkpen & & & & 110 & & & & & & & & & & & & & & & & & 110 & $100 \%$ \\
\hline StepOver blueM Pad & & & & 386 & & & & & & & & & & & & & & & & & 386 & $100 \%$ \\
\hline \begin{tabular}{|l|} 
Wacom SCREEN \\
\end{tabular} & & & & & & & 158 & & & & & & & & & & & & & & 158 & $100 \%$ \\
\hline Wacom USB SCREEN & 8 & & & & 202 & & 2986 & & & & & & & & & & & & & & 3196 & $99.75 \%$ \\
\hline Wacom USB NO ANGLE & & & & & & & & & 42 & 6 & & & & & 23 & & & & & & 71 & $100 \%$ \\
\hline Wacom Intuos 2 SCREEN & & & & & & 14 & & & & & & & & & & & & & & & 14 & $100 \%$ \\
\hline Wacom Intuos 2 NO ANGLE & & & & & & & & & 6 & & & & & & & & & 20 & 11 & & 37 & $16.22 \%$ \\
\hline Wacom Volito SCREEN & & & & & & 4 & & & & & & & & & & & & & & & 4 & $100 \%$ \\
\hline
\end{tabular}


Table. 8. Devices in regard to their true positive and false positive determination results

\begin{tabular}{|l|l|l|l|}
\hline Device & Correct Identification Ratio & False Identification Ratio & Non Classification Ratio \\
\hline Palm & 1 & 0 & 0 \\
\hline PocketPC & 1 & 0 & 0 \\
\hline StepOver +Pad & 1 & 0 & 0 \\
\hline StepOver +Pad Inkpen & 1 & 0 & 0 \\
\hline StepOver +Pad NO PRESS & 1 & 0 & 0 \\
\hline StepOver blueM Pad & 1 & 0 & 0 \\
\hline Touch EIZO & 1 & 0 & 0 \\
\hline Wacom Intuos 2 Inkpen & 1 & 0 & 0 \\
\hline Wacom Intuos SCREEN & 1 & 0 & 0 \\
\hline Wacom SCREEN & 1 & 0 & 0 \\
\hline Wacom USB NO ANGLE & 1 & 0 & 0 \\
\hline Wacom Volito SCREEN & 1 & 0 & 0 \\
\hline Logitech IO-Pen & 0.992596375 & 0 & 0.007403625 \\
\hline Wacom Volito & 0.982683983 & 0 & 0 \\
\hline Wacom Cintiq 15 & 0.975844949 & 0.017316017 & 0.011170527 \\
\hline Toshiba Portégé Tablet-PC & 0.956159308 & 0.012984533 & 0.026860143 \\
\hline Wacom & 0.928363988 & 0.016980549 & 0.071636012 \\
\hline Wacom USB SCREEN & 0.917496871 & 0 & 0.82503129 \\
\hline Wacom Intuos 2 & 0.715147858 & 0.038020519 \\
\hline Aiptec Hyperpen 8000 & 0.517241379 & 0 \\
\hline Wacom USB & 0.441728763 & 0.246831623 & 0.002086438 \\
\hline Touch & 0.367963976 & 0.482758621 & 0.008684464 \\
\hline Wacom Intuos 2 NO ANGLE & 0.162162162 & 0.556184799 & 0 \\
\hline
\end{tabular}

In general, as it can be seen in column OPC0, relatively few non-classifications have been observed all together. 12 out of the 23 tested pen digitizers $(52.17 \%$ ) could be correctly identified in all cases (Correct Identification rate of $100 \%$ ). Another 3 tested pen digitizers (Wacom USB SCREEN, Wacom, and Logitech IO-Pen) are identified with almost 100\% accuracy. Here, only non-classification outliers but no false identifications negatively influenced the result (i.e. False Identification Rate yields 0). Depending on the total number of tested pen digitizers of a specific type and the corresponding number of outliers, the result ranges between $92.84 \%$ and $99.75 \%$. Based on approximately 40,000 tested samples, an average identification rate of $87 \%$ could be achieved by the presented concept across all tablets.

Wacom USB, Touch, and Aiptec Hyperpen 8000 seem to be problematic, since they are only providing a low accuracy of identification in the range of $36.80 \%-51.72 \%$. In other words, at least every second device could not be identified. A possible explanation for this phenomenon could be the pressure resolution. These devices are supposed to provide a maximum pressure quantization of 512 levels (Aiptec Hyperpen 8000) or 1024 levels (Wacom USB, Touch). Regarding Aiptec Hyperpen 8000, a pressure quantization less than half the maximum pressure quantization of $512(<256)$ can be observed in some samples. Regarding Wacom USB and Touch, some samples in the test set provide a zero pressure quantization. Apparently for these two tablet categories, an erroneous data acquisition resulted in inconsistent data. With respect to the low identification rates, extracting such samples would significantly increase the identification accuracy.

Furthermore, the Wacom Intuos 2 NO ANGEL device shows a poor accuracy of identification with only $16.22 \%$. Here, no angle information is supposed to be included in the samples, but some samples contain a detected angle value nevertheless. Neglecting theses samples, would also improve the identification accuracy.

Generally, all StepOver devices seem to have a total accurate identification rate of $100 \%$. Three of these devices ( + Pad, + Pad Inkpen and blueM Pad) belong to the same output group which indicates an impossible discrimination of StepOver devices by using the presented approach, which fits to our expectations, as all three tablets use the same sensor technology. StepOver +Pad NO PRESS digitizers are also based on this technology, but samples in this category have been collected neglecting the pressure information in the signals. This category has also shown $100 \%$ correct identification rates, but has been classified in a separate output class, OPC7.

Finally, other devices with zero pressure quantization and low spatial resultion such as Palm, Touch Eizo, and Wacom USB SCREEN cannot be differentiated. Therefore, considering further statistical parameters and enhancing the presented approach is subjective of ongoing research. 


\section{Conclusion and Future Work}

In this paper, a new approach has been presented to identify pen-based digitizer devices. This has been done with the background of introducing the new research area of sensometrics, the connector of biometrics and forensics. From approximately 40,000 handwritten samples taken from 23 different digitizer tablets, specific features for classification have been extracted and a feature vector classification system based on a decision tree model has been implemented and experimentally evaluated. The decision tree classification resulted in 19 output groups for the 23 devices.

For each digitizer type, we have divided the database into two disjoint partitions: the analyzing partition for development of the decision tree model and the output classes (OPC) and a disjoint testing partition for our subsequent evaluation. Thus, an equal number of samples is represented in either of the sets for each digitizer type. To our knowledge this approach constitutes the first approach to identify a pen digitizer, i.e. to apply sensometric schemes to this category of sensors.

In our evaluation we have shown that 12 out of the 23 devices could be identified with $100 \%$ accuracy and another six showed correct identification rates of over $90 \%$. However, as already be outlined in section 6 , two major restrictions of your concept have been observed during testing: First, pen digitizers with the same sensor technology could not be uniquely identified. Second, all low resolution devices resulted in high false identification rates. But in the context of mobile multimedia those low resolution devices are playing a major role as they are mainly applied in that area.

Taking this as a basis for future and ongoing research we put much effort in enhancing the existing decision tree model by testing statistical classification methods in comparison to our developed decision tree approach. Additionally, we are focussing on finding further features to also uniquely identify different instances of devices having identical sensor technology, in analogy to the results reported for CCD (Charge Coupled Devices) in [3] or [4].

\section{ACKNOWLEDGEMENTS}

This work has been partly supported by the EU Network of Excellence SIMILAR (Proposal Reference Number: FP6507609). The contents of this publication are the sole responsibility of the authors and can in no way be taken to reflect the views of the European Union. Our special thanks belong to Vissapragada Venkata Ramana Murthy doing the analysis of our data set, the implementation and evaluation of the decision tree.

\section{REFERENCES}

1. 8th Sensometrics Meeting, Imagine the senses, 2 - 4 August 2006, Ås, Norway, URL:

http://www.sensometric.org/pages/meet.htm, last requested January 2007.

2. C. Vielhauer: Biometric User Authentication for IT Security, Advances in Information Security. Vol. 18, Springer, New York ISBN: 0-387-26194-X, 2006.

3. J. Lukas, J. Fridrich and M. Goljan, "Determining Digital Image Origin Using Sensor Imperfections," Proc. SPIE Electronic Imaging San Jose, CA, January 16-20, 249-260 (2005).

4. J. Fridrich, J. Lukas and M. Goljan, "Digital Camera Identification from Sensor Noise," IEEE Transactions on Information Security and Forensics, June 2006, Vol. 1(2), 205-214 (2006).

5. A. Oermann, A. Lang, J. Dittmann, "Verifyer-Tupel for Audio-Forensic to Determine Speaker Environment," City University of New York (Organizer): Multimedia and Security, MM \& Sec'05, Proceedings ACM, Workshop New York, NY, USA, August 1-2, 57-62 (2005).

6. A. K. Mikkilineni, P.-J. Chiang, G. N. Ali, G. T. C. Chiu, J. P. Allebach, and Edward J. Delp III, "Printer identification based on graylevel co-occurrence features for security and forensic applications," In: Proceedings of the SPIE International Conference on Security, Steganography, and Watermarking of Multimedia Contents VII, SSWMC, San Jose, California, USA, January 1720, vol. 5681, 430-440 (2005).

7. A.K. Mikkilineni, O. Arslan, P.-J. Chiang, R.M. Kumontoy, J.P. Allebach, G.T.-C. Chiu, et al., "Printer forensics using svm techniques," In: Proceedings of the IS\&T's NIP21: International Conference on Digital Printing Technologies, Baltimore, MD, October 2005, Vol. 21, 223-226 (2005).

8. N. Khanna, A. K. Mikkilineni, A. F. Martone, G. N. Ali, G. T.-C. Chiu, J. P. Allebach, and E. J. Delp, "A survey of forensic characterization methods for physical devices," In: Proceedings of the 6th Digital Forensics Research Workshop (DFRWS), Lafayette, Indiana, August 2006, 17-28 (2006). 
9. R. Plamandon and G. Lorette, "Automatic Signature Verification and Writer Identification - the State of the Art," Pergamon Press plc., Pattern Recognition, 22, Vol. 2, 107-131 (1989).

10. F. Leclerc and R. Plamondon, "Automatic Verifictaion and Writer Identification: The State of the Art 1989-1993," International Journal of Pattern Recognition and Artificial Intelligence, Vol. 8, 643-660 (1994).

11. J. Gupta and A. McCabe, "A Review of Dynamic Handwritten Signature Verification," Technical report at James Cook University, Australia, (1997).

12. C. Vielhauer, R. Steinmetz, and A. Mayerhöfer, "Biometric Hash based on Statistical Features of Online Signatures," In: Proceedings of the IEEE International Conference on Pattern Recognition (ICPR), Canada, Vol. 1, 123-126 (2002).

13. The Culture Tech Project, Cultural Dimensions in digital Multimedia Security Technology, a project funded under the EU-India Economic Cross Cultural Program, http://amsl-smb.cs.uni-magdeburg.de/culturetech/, last requested January 2007. 